\title{
Art in hospital: is it possible?
}

\author{
Anna Belfiore, Vincenzo Ostilio Palmieri, Giuseppe Palasciano
}

Clinica Medica A. Murri, Policlinico, Bari, Italy

\begin{abstract}
This review explores the relationship between art and hospital, highlighting how art in hospitals can become a crucial element of the clinical governance to enhance the well being of the patients. To support this position, the authors report the experience derived from the program of Art and Culture in Hospital, which has been developed for about 10 years at the Clinic of Internal Medicine A. Murri Policlinic of Bari.
\end{abstract}

\section{Art in hospital}

The concepts of art and hospital appear at first glance to be two irreconcilable entities. Art refers to the emotional, creative and introspective level, hospital refers to the technological and scientific level. To understand the full meaning of the relation art-hospi$\mathrm{tal}$, it is appropriate to re-evaluate the concept of health and disease.

The classical idea of health, understood as the $a b$ sence of disease, has been modified by the World Health Organization (WHO) in agreement with a holistic view of health. The WHO defines holistic health as:

viewing man in his totality within a wide ecological spectrum, and... emphasizing the view that ill health or disease is brought about by an imbalance, or disequilibrium, of man in his total ecolog-

Correspondence: Anna Belfiore, Clinica Medica A. Murri, Policlinico, piazza G. Cesare, 70100 Bari, Italy.

Tel. +39.340.7935720.

E-mail: belfiore.murri@gmail.com

Key words: medicine, art, hospital.

Acknowledgements: the authors would like to thank Mrs. Ann Pilkington, for her support in reviewing the English text.

Conflict of interests: the authors declare no potential conflict of interests.

Received for publication: 9 March 2014.

Accepted for publication: 17 March 2014.

This work is licensed under a Creative Commons Attribution NonCommercial 3.0 License (CC BY-NC 3.0).

(C) Copyright A. Belfiore et al., 2015

Licensee PAGEPress, Italy

Italian Journal of Medicine 2015; 9:89-91

doi:10.4081/itjm.2014.503 ical system and not only by causative agent and pathogenic evolution. ${ }^{1}$

In this context, health and disease are not two distinct entities, but they have both relative and subjective meanings, strongly influenced by the cultural contest and the psychological state of the subject.

We can consider, for example, chronic cardiovascular diseases, which are now the leading cause of death in western countries. These diseases are frequently associated with psychosocial problems such as depression and states of anxiety, contributing to negative outcomes. ${ }^{2,3}$ Several observations have shown that the introduction of creative activities in an ideal therapeutic program reduces stress and depression and improve the outcome of chronic disease. There is growing international agreement that participation in the creative arts can be beneficial for health. The creative therapies most often used are: music, visual arts and expressive writing. ${ }^{4}$

\section{The new hospital}

The new concept of health and disease has influenced the way of understanding the hospital-structure, where the art of healing is practiced. Attention to physical environment as an important variable in the care process has challenged the traditional setting of hospitals as depersonalized and depersonalizing places. The hospital should not be considered as a place of suffering and disease, but as the best place to promote the healing process. Architecture plays a vital role in creating facilities for patients without depriving them of all their cultural and emotional components. The very name of the hospital reflects its essential function; the adjective hospitalis means: spiritually warm and comfortable. The architecture of hospitals contributes to well being and recovery of patients.

In the Declaration of Ottawa in 2004, the WHO emphasized the importance of the environment in the healing process. Recently, the International Academy of 
Design and Health, during the International Congress of 2011, has focused attention on the importance of architecture in health promotion. Hospitals should be designed as places that can affect health positively. Today hospital designers are trying to evolve hospitals beyond their sterile décor. A hospital consistent with the needs of the patients should include shelters, natural green spaces, recreational areas, waiting areas, removal of architecture barriers. In addition the use of color, of lighting and of artwork can give patients more positive experiences. The use of color in the hospital is more and more frequent. Introducing the right colors in different hospital environments can positively influence the level of stress and mood of the patients.

In Italy, an example of hospital architecture that has realized these elements is the Meyer Children's Hospital in Florence. The hospital is a pleasant and functional building with children's play area and outdoor play area as well. The wards are decorated in cheerful colors such as orange and with art works, which are appealing to children. All spaces are designed in favor of the children and their families. The aim of the hospital is respect for the human and cultural dimension of the children along with health care excellence.

\section{Art works in hospital}

The presence of works of art in the hospital does not only have the function of mere decoration. The work of art, painting, sculpture or murals, are designed to counteract the feelings of alienation and isolation created by the disease and the hospital. The creation of a harmonious space in hospital can help reflection on the particular moment of the disease and encourage the search for personal resources to face up to it.

Beauty in this context takes again its original meaning of salvation, justice, moral category.

This observation inevitably leads us to consider what exactly is medicine, always suspended between science and practice. As a practice, medicine is interested in man who has existence (in the philosophical sense of problems, determination and freedom), consciousness and language, and who lives in a cultural and relational contest. Man remains such even when he becomes a patient, and art works in this direction. However, art in hospital has a dual function. It acts on the patient and also on the doctor working as a facilitator of communication, reducing stress and symptoms of burnout that are associated with a profession that provides continuous contact with disease.

In the hospital, art can become the vehicle to understand the patient. The study of art and literature should be included in some way in the curricula of Medicine, to train doctors not only to be professionally and scientifically valid, but also to render them capa- ble of a deeper understanding of the patient as a whole. Literature, painting, sculpture, can provide different interpretations of the complex reality of the human subject, often assessed only through the point of view of the disease in the hospital. Art becomes a way of knowledge. The great writers, such as Thomas Mann, Manzoni, Dostoevsky, and many others, who have addressed the problem of disease and described the feelings of patients, can be of great help. In this regard, Vito Cagli writes in his book Malattie come racconti: ...their sensitivity can provide a valuable path to lead us to understand exactly what the uproar of hurry, of technology, of the importance of objectivity, threaten to obscure. ${ }^{5}$

In this contest the connection between art and medicine becomes understandable. This correlation facilitates the process of humanization of the hospital, enhances the individuality of the patient, improves the quality of their stay in hospital and can act as a facilitator to healing.

The idea of art in hospital has developed in different geographical areas. In Europe, France has been more attentive to the new needs of the health care fields. The association L'art dans la cite, which aims to introduce contemporary artworks in hospitals, has been active for several years in France. The specific goal of this association is the creation of artworks directly in hospitals, where artists work in partnership with patients. Participation in artistic activity on the part of patients (often the young patients of pediatric clinics) improves the quality of life, provides an alternative to the passivity of hospital care and therefore gives a more human dimension to the hospital.

Currently several Italian hospitals have included events and artworks in the hospital setting.

The question is whether these interventions can have positive effects in the healing process. Systematic and controlled studies, which analyze the therapeutic effect of art, have emerged only recently. A recent article by Ann Drahota, published by the Cochrane Library, has analyzed the effect of health facilitators can offer in terms of recovery. ${ }^{6}$ The author has researched the works only on the major search engines, taking into account only those observations, all carried out in hospital for adults, where they were shown the effects on some parameters of health. The author defined positive distracters as elements of the sensory environment that included: aromas/scents; viewing artwork; viewing performance art; audiovisual distractions (television/video); decoration (color of walls); music; access to nature (gardens, window views or indoor plants). The results obtained from this original meta-analysis confirmed that music could improve patient-reported outcomes such as anxiety.

Another Italian observation made at the university of Tor Vergata, has highlighted the importance of ac- 
tive participation or interest in various forms of art. ${ }^{7}$ The authors have compared the quality of life of the admirers of art with that of people who have never been interested in any form of art, within a group of 192 people affected by strokes. The 105 people who were familiar with art enjoyed better health and, after the stroke, had begun to walk more easily, and in general were happier, less anxious and depressed. We could conclude that art can make our brain more plastic after an acute event such as a stroke. It is known that listening to music stimulates the pleasure center and promotes the release of dopamine, which triggers a circuit of gratification with the production of endorphins. It was also observed that music can reduce neuronal activity and states of anxiety, and can help to restore the function of the immune system by way of the amygdale and hypothalamus. ${ }^{8}$ The anti-stress effects of music have been evaluated in several studies, in particular its sedative influence and harbinger of pleasant sensations was observed mainly in patients suffering from chronic pain or heart attack, for which the stress reduction is crucial.

At the Clinic of Internal Medicine A. Murri Policlinic of Bari, a program of Art and Culture in Hospital has been developed for about 10 years, which has introduced elements of art and artistic performances in the hospital to improve the well being of the patients and personnel. The department, the hospital rooms, the waiting rooms have been brightened up with numerous art posters, selecting those works where color and natural environments are dominant. A concert is organized each month and the patients with their relatives and caregivers can listen to the music program, happily forgetting the fact that they are patients or actually in hospital. The concerts range from classical to popular music, from instrumental music to opera. In the afternoons of music the wards change appearance. The patients have the sensation of going to the theater. During the concerts they meet their own doctors in the same role of spectators and can easily express their feeling of aversion or of acceptance of the disease. The concerts are an opportunity for artistic improvisation on the part of older patients, always happy to show off their singing skills. In the days following the concert our patients report feeling well in the clinic and express their appreciation of the initiative. We favored music amongst the different artistic expressions, because it has a common language, it does not need interpreters or translators and speaks directly to the deepest chords of each subject.

This is what one patient, both a music lover and spectator at a musical afternoon in the clinic wrote:

A pale existential prelude by Chopin, well presented and performed, felt emotionally by the patients, would lead them to a stronger, more dynamic vision of their illness, strengthening their ability to deal with it. ${ }^{9}$

\section{References}

1. World Health Organization. Traditional medicine: proposed programme budget for the financial period 1981. Geneva: World Health Organization; 1978.

2. Lewis TT, Everson SA, Powell LH, et al. Chronic exposure to everyday discrimination and coronary artery calcification in African-American women: the SWAN heart study. Psychosom Med 2006;68:362-8.

3. McEwen BS, Stellar E. Stress and the individual: mechanisms leading to disease. Arch Intern Med 1993;153: 2093-101.

4. Stuckey HL, Nobel J. The connection between art, healing and public health: a review of current literature. Public Health 2010;100:254-63.

5. Cagli V. Malattie come racconti. Roma: Armando Editore; 2004.

6. Drahota A, Ward D, Mackenzie H, et al. Sensory environment on health-related outcomes of hospital patients. Cochrane Database Syst Rev 2012;(3):CD005315.

7. Vellone E, Alvaro R, Caramia M. Stroke survivors who like art have a better quality of life than those who do not. Eur J Cardiovasc Nurs 2012;11:S8.

8. Angelucci F, Ricci E, Padua P, et al. Music exposure differentially alters the levels of brain-derived neurotrophic factor and nerve growth factor in the mouse hypothalamus. Neurosci Lett 2007;429:152-5.

9. Papapietro G. La politica del dialogo. Bari: Progedit; 2007. 